\title{
Therapeutic Effect of Korean Red Ginseng Extract on Infertility Caused by Polycystic Ovaries
}

\author{
Ji-Hun Jung ${ }^{1 \#, ~ H y u n ~ T a e ~ P a r k ", ~ T a k ~ K i m ², ~ M o o n ~ J i n ~ J e o n g ~}{ }^{3}$, Sung Chul Lim ${ }^{4}$, Seung Yeol Nah ${ }^{5}$, \\ Ik-Hyun Cho ${ }^{6}$, Soo Hyun Park ${ }^{1}$, Seong Soo Kang ${ }^{1}$, Chang Jong Moon ${ }^{1}$, Jong Choon Kim ${ }^{1}$, \\ Sung Ho Kim ${ }^{1}$, and Chun Sik Bae ${ }^{1 *}$ \\ ${ }^{1}$ Department of Veterinary Surgery, Chonnam National University College of Veterinary Medicine, Gwangju 500-757, Korea \\ ${ }^{2}$ Department of Obstetrics and Gynecology, Korea University College of Medicine, Seoul 136-705, Korea \\ ${ }^{3}$ Department of Oral Histology, Chosun University School of Dentistry, Gwangju 501-825, Korea \\ ${ }^{4}$ Department of Pathology and Research Center for Resistant Cells, Chosun University School of Medicine, \\ Gwangju 501-825, Korea \\ ${ }^{5}$ Department of Physiology, Konkuk University College of Veterinary Medicine, Seoul 143-701, Korea \\ ${ }^{6}$ Department of Anatomy, College of Oriental Medicine and Institute of Oriental Medicine, Kyung Hee University, \\ Seoul 130-701, Korea
}

Polycystic ovarian syndrome (PCOS) is a very common endocrine disorder in women of reproductive age. Nerve growth factor (NGF) may be involved in the pathogenesis of PCOS. In this study, we investigated the effect of Korean red ginseng extract (KRGE) on the ovarian morphology and NGF expression in an estradiol valerate (EV)-induced rat model. Polycystic ovaries were induced by a single intramuscular injection of estadiol valerate (4 mg, dissolved in sesame oil) in adult cycling rats. KRGE was administered orally ( $200 \mathrm{mg} / \mathrm{kg}$ body weight/day) for 60 consecutive days, beginning 60 days after the induction. Ovarian morphology was almost normalized and NGF was normalized in the EV+KRGE group. KRGE lowered the high numbers of antral follicles and increased the number of corpora lutea in the polycystic ovaries. The results are consistent with a beneficial effect of KRGE in the treatment of PCOS.

Keywords: Panax ginseng, Polycystic ovary syndrome, Estradiol valerate, Pathophysiology, Ginseng extract

\section{INTRODUCTION}

Polycystic ovarian syndrome (PCOS) is a highly prevalent heterogeneous syndrome of clinical and/or biochemical androgen excess, ovulatory dysfunction, and polycystic ovaries $[1,2]$. The pathophysiology responsible for the development of polycystic ovaries has puzzled researchers for many years and proven very dif- ficult to define. Polycystic ovaries are likely associated with ovarian hyperandrogenism, hyperinsulinemia, and altered intraovarian paracrine signaling $[3,4]$. Among the plethora of peptides and steroids produced by the ovary, androgens appear to play a key role in the etiology of PCOS $[1,5]$. However, they may not be a causative factor

\footnotetext{
(c) This is an Open Access article distributed under the terms of the Creative Commons Attribution Non-Commercial License (http://creativecommons.org/licenses/by-nc/3.0/) which permits unrestricted non-commercial use, distribution, and reproduction in any medium, provided the original work is properly cited.
}

Received 14 Mar. 2011, Revised 06 May 2011, Accepted 06 May 2011

\#These two authors contributed equally to this work.

*Corresponding author

E-mail: csbae210@chonnam.ac.kr

Tel: +82-62-530-2876, Fax: +82-62-530-2809 
of polycystic ovaries because this morphology is not associated with changes in androgen production $[6,7]$.

Evidence exists that supports the association of PCOS with increased sympathetic activity [8]. The role of the sympathetic nervous system in the pathophysiology of PCOS is supported by the denser innervation of catecholaminergic nerve fibers in polycystic ovaries compared to cyclic ovaries [9]. Rats injected with a single dose of estradiol valerate (EV) develop an anovulatory condition that resembles human PCOS $[10,11]$. Using this rodent model, we demonstrated that higher activity of the ovarian sympathetic nervous system in a polycystic ovaries rat is related to an increased synthesis of ovarian nerve growth factor (NGF), a neurotrophin produced by nonneuronal ovarian cells $[12,13]$. Studies in rats have raised the possibility that the deranged production of NGF may contribute to the development of polycystic ovaries. Additionally, several human studies have indicated that an excess of intraovarian NGF contributes to human polycystic ovaries [14].

Previously, we reported that Korean red ginseng extract (KRGE) attenuates the histologic findings of polycystic ovaries and NGF expression in the ovaries of EV-induced PCOS rats when KRGE is administered beginning a day after EV injection [13]. EV-induced polycystic ovaries could be detected approximately 60 days after EV injection; activation of the sympathetic innervation to the ovary precedes the formation of polycystic ovaries by at least a month $[11,15]$. Therefore, we cannot definitively conclude that these results reflected the effects of KRGE on established polycystic ovaries. It is likely that once polycystic ovaries are maintained in adulthood following their establishment in adolescence [15]. The aim of the present study was to investigate the effect of KRGE on ovarian sympathetic innervation in rats with fully developed polycystic ovaries induced by EV.

\section{MATERIALS AND METHODS}

\section{Animals}

Sprague-Dawley female rats $(n=21)$ weighing 190 to 210 $\mathrm{g}$ and with regular 4-day estrous cycles were purchased from Samtako (Osan, Korea). The rats were quarantined and acclimated for 1 week prior to use. The Institutional Animal Care and Use Committee at Chonnam National University approved the protocols used in this study, and the animals were cared for in accordance with the Guidelines for Animal Experiments. The animals were housed in a room that was maintained at $23 \pm 2^{\circ} \mathrm{C}$, relative humidity of 50 $\pm 5 \%$, artificial lighting from $08: 00$ to $20: 00 \mathrm{~h}$ and with 13 to 18 air changes per hour. The animals were housed polycarbonate cages and were given tap water and commercial rodent chow (Samyang Feed, Daejeon, Korea) ad libitum. The animals were blindly randomized into an EV control group ( $n=7), \mathrm{EV}+$ saline control group ( $1 \mathrm{~mL} / \mathrm{kg}$ saline, $n=7)$ and $\mathrm{EV}+\mathrm{KRGE}$ group (200 $\mathrm{mg} / \mathrm{kg}$ KRGE orally everyday for 60 days, beginning 60 days after the EV injection; $n=7$ ).

\section{Induction of polycystic ovaries}

Twenty one rats received a single $4 \mathrm{mg}$ intramuscular injection of EV (Sigma-Aldrich, St. Louis, MO, USA) in $0.2 \mathrm{~mL}$ of sesame oil (Sigma-Aldrich) to induce polycystic ovaries, as previously described [13]. To obtain fully developed polycystic ovaries, duration of 60 days after the injection was chosen.

\section{Korean red ginseng extract}

KRGE was manufactured from the roots of a 6-yearold fresh Panax ginseng and provided by the Korea Ginseng \& Tobacco Central Research Institute (Daejeon, Korea). The provided KRGE contained 11 glycosides as known ginsenosides: $\mathrm{Rb}_{1}(15.82 \%), \mathrm{Rb}_{2}(7.79 \%)$, $\operatorname{Rc}(8.06 \%), \operatorname{Rd}(7.57 \%), \operatorname{Re}(3.21 \%), \operatorname{Rf}(4.72 \%), \operatorname{Rg}_{1}$ (1.91\%), $\operatorname{Rg}_{2}(22.08 \%), \operatorname{Rg}_{3}(24.06 \%), \mathrm{Rh}_{1}(4.63 \%)$, and $\mathrm{Rh}_{2}(0.15 \%)$. The KRGE was dissolved in saline and administered orally.

\section{Ovarian morphology}

At the end of the experiments, all rats were anesthesized using an intraperitoneal injection of chloral hydrate $(500 \mathrm{mg} / \mathrm{kg})$ prior to treatment with a fixative of $4 \%$ paraformaldehyde- $4 \%$ sucrose in $0.1 \mathrm{M}$ sodium cacodylate buffer ( $\mathrm{pH}$ 7.4). The dissected ovaries were placed in the same fixative overnight at $4^{\circ} \mathrm{C}$ and embedded in paraffin. Samples were sectioned at a thickness of $4 \mu \mathrm{m}$ and stained with hematoxylin and eosin. Follicles containing an oocyte with a nucleus were counted and analyzed by an experienced pathologist. If ovum degeneration or at least one pyknotic granulosa cell was seen, the follicle populations were classified as atretic, otherwise they were classified as healthy. Morphological characteristics of follicular atresia were scattered pyknotic nuclei in the granulosa cell layer, detachment of the granulosa cell layer from the basement membrane, fragmentation of the basal lamina and the presence of cell debris in the antrum of the follicle.

\section{Western blot analysis}

Protein samples (30 $\mu \mathrm{g} / \mathrm{lane})$ were separated by $12 \%$ 
sodium dodecyl sulfate-polyacrylamide gel elecrophoresis (SDS-PAGE) for the detection of NGF- $\beta$. Protein samples were diluted in SDS-PAGE sample buffer and boiled for $10 \mathrm{~min}$ before being loaded onto the gels. After electrophoresis, the resolved proteins were transferred to a nitrocellulose membrane in transfer buffer $(20 \%$ methanol, $25 \mathrm{mM}$ Tris, $40 \mathrm{mM}$ glycine, $\mathrm{pH}$ 8.3). The membrane was blocked using 5\% non-fat dry milk and blotted with a 1:2,500 dilution of anti-rabbit NGF antibody (Abcam, Cambridge, MA, USA), followed by a 1:10,000 dilution of goat antirabbit-IgG antibody (Santa Cruz Biotechnology, Santa Cruz, CA, USA). Blots were washed in phosphate-buffered saline-Tween 20. The bound antibody was detected by enhanced chemiluminescence (Amersham Pharmacia, Buckinghamshire, UK). Protein loading was normalized to a 1:2,500 dilution of $\beta$-actin (Sigma-Aldrich). The relative density of the bands was densitometrically analyzed using Sciencelab Image Gauge ver. 3.12 (Fujifilm, Tokyo, Japan).

\section{Reverse transcription-polymerase chain reaction}

Total RNA was extracted from frozen homogenized ovary tissue using TRI reagent (Molecular Research Center, Cincinnati, OH, USA). Accupower RT premix with ExTaq (TaKaRa Bio, Seoul, Korea) was used for first strand cDNA synthesis and polymerase chain reaction (PCR) amplication, according to the manufacturer's instructions. cDNA synthesis was achieved by incubating template RNA $(1 \mu \mathrm{g})$ at $70^{\circ} \mathrm{C}$ and reverse transcriptase mix in a reaction volume of $20 \mathrm{~mL}$ for $60 \mathrm{~min}$ at $42^{\circ} \mathrm{C}$, then for $5 \mathrm{~min}$ at $94^{\circ} \mathrm{C}$ in a PCR Thermal Cycler GP (TaKaRa Bio, Shiga, Japan). PCR was carried out using the primer pairs derived from the published rat NGF- $\beta$ sequence (forward, 5'-CTT CAG CAT TCC CTT GAC AC-3'; and reverse, 5'-TGA GCA CAC ACA CGC AGG C-3') [16] and primers for rat glyceraldehyde3-phosphate dehydrogenase (GAPDH) (forward, 5'CCA TGG AGA AGG CTG GG-3'; and reverse, 5'-CAA
AGT TGT CAT GGA TGA CC-3'). The housekeeping gene GAPDH was used as an internal control to determine the relative concentration of NGF- $\beta$. Amplified PCR products (NGF- $\beta, 592$ bp; GAPDH, 199 bp) were size-fractionated by electrophoresis through a $1.5 \%$ agarose gel and stained with ethidium bromide $(0.2 \mu \mathrm{g} / \mathrm{mL})$. The DNA bands were visualized and quantified by densitometry and expressed as the ratio of the densitometric analysis for each target gene normalized to the GAPDH control. The density of each band was quantified as described above.

\section{Statistical analysis}

Data presented are the mean values from three animals \pm SD. Data are representative of three independent experiments. Statistical analysis of the data was performed using a Student's $t$-test with a Bonferroni correction for analysis of multiple comparisons. The differences were considered significant at $p<0.05$.

\section{RESULTS}

\section{Histological findings}

Ovaries in the EV control group exhibited numerous cystic follicles in the ovarian cortex (Fig. 1A). In the $\mathrm{EV}+$ saline control group, cystic follicles were decreased in number (Fig. 1B). The numbers of corpora lutea and corpora albicantia in the EV+KRGE group were significantly higher than the EV control group, while the number of cystic follicles was lower (Fig. 1C). More growing secondary follicles were evident, compared with the EV control group.

\section{NGF changes after EV injection and response to KRGE administration}

The ovarian NGF mRNA expression was significantly increased in the EV-treated control group. In the EV group receiving KRGE, NGF mRNA was significantly

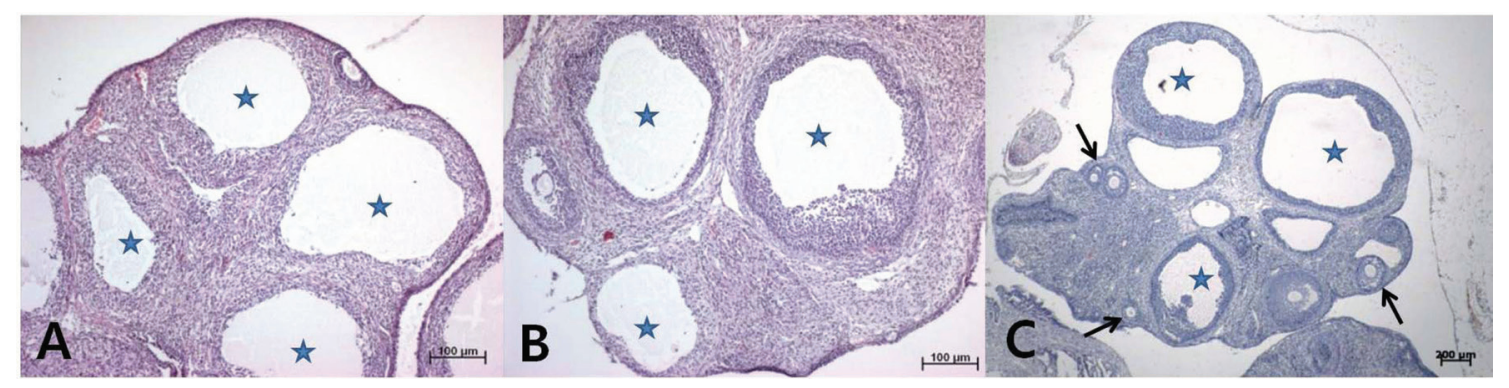

Fig. 1. Morphological changes of the ovary. (A) Estradiol valerate (EV) group: numerous cystic follicles (asterisks) were identified in the ovarian cortex. (B) EV+saline control group: cystic follicles (asterisks) were decreased in number. (C) EV+Korean red ginseng extract group: cystic follicles (asterisks) were decreased in number. Some normal follicles (arrows) were noted too (H\&E, x40). 

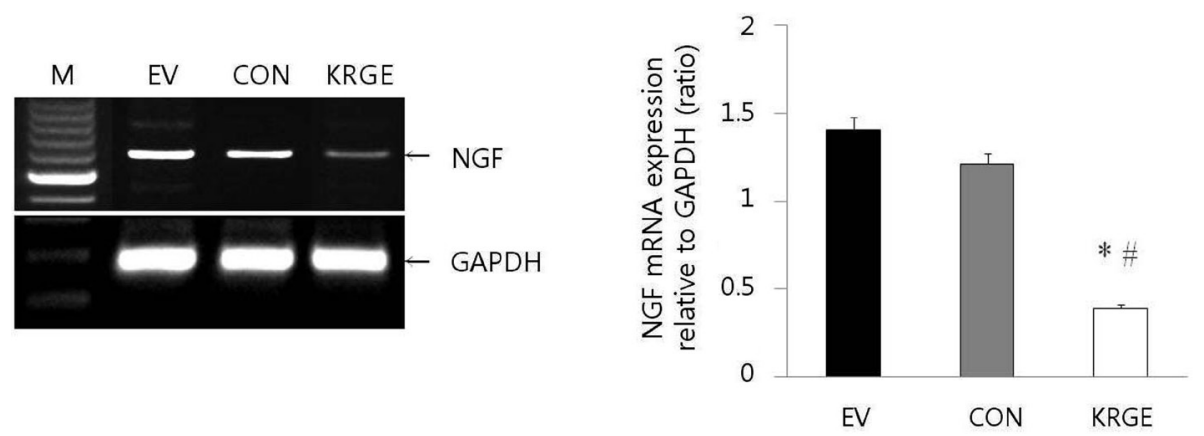

Fig. 2. Ovarian nerve growth factor (NGF) mRNA levels in control and polycystic ovaries rats treated with Korean red ginseng extract (KRGE). NGF mRNA increased in the estradiol valerate (EV)-treated polycystic ovaries group. An increase in mRNA expression was reversed by KRGE administration in polycystic ovaries rats. Values are given as mean \pm SD normalized to glyceraldehyde-3-phosphate dehydrogenase (GAPDH). A representative reverse transcription-polymerase chain reaction is shown at left. M, DNA marker; EV, EV control group; CON, EV plus saline; KRGE, EV plus KRGE. ${ }^{*} p<0.05$ vs. EV group; ${ }^{*} p<0.05$ vs. CON group.
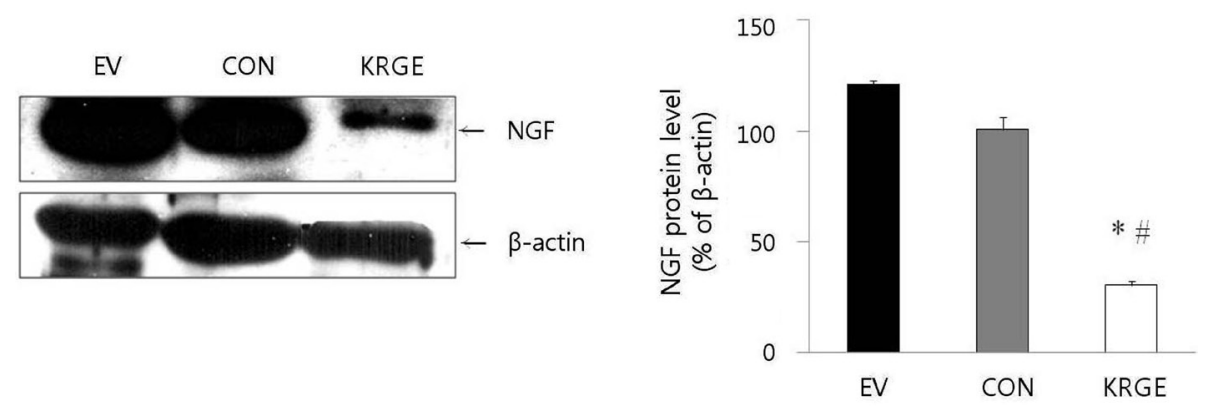

Fig. 3. Ovarian nerve growth factor (NGF) protein levels in control and polycystic ovaries rats treated with Korean red ginseng extract (KRGE). Protein level of NGF was higher in the estradiol valerate (EV)-treated polycystic ovaries group and KRGE treatment reversed the stimulatory effect of EV. Values are mean \pm SD normalized for $\beta$-actin. A representative Western blot is shown at left. EV, EV control group; CON, EV+saline; KRGE, EV+KRGE. * $p<0.05$ vs. EV group; ${ }^{*} p<0.05$ vs. CON group.

depressed compared with the rats exhibiting EV-induced polycystic ovaries $(p<0.05)$ (Fig. 2). Likewise, the concentration of ovarian NGF protein was significantly higher in the EV group, and daily KRGE administration resulted in significantly lower concentration of ovarian NGF protein in the rats with polycystic ovaries receiving KRGE than in the EV control group $(p<0.05)$ (Fig. $3)$. The ovarian concentration of NGF protein in the EV control group and the polycystic ovaries group receiving saline were similar.

\section{DISCUSSION}

We previously demonstrated the effects of KRGE on the development of polycystic ovaries by administering KRGE beginning a day after EV injection [13]. The present results show that KRGE can attenuate the sympathetic marker in terms of ovarian NGF, even after polycystic ovaries are fully developed. This suggests that KRGE could have an impact on established polycystic ovaries.

PCOS is the most common endocrine disorder in women of reproductive age. A huge amount of healthcare resources have been used for PCOS-related disorders. Several pharmacological agents, including oral contraceptives and metformin have been used for the restoration of menstruation and treatment of hyperandrogenism $[17,18]$. However, it remains controversial whether pharmacological agents can modulate polycystic ovaries morphology [19-21]. Moreover, the effects of these agents are temporary, and clinical manifestations of PCOS reappear after cessation of the medication. Thus, it is clinically important to evaluate whether KRGE has an impact on rat polycystic ovaries, which may reflect an impact on human polycystic ovaries.

The presence of polycystic ovaries has recently been included as a key element for the diagnosis of PCOS, 
leading to two different sets of diagnostic criteria [22,23]. Polycystic ovaries can be observed during pubertal development and in patients with hypothalamic amenorrhea or hyperprolactinemia [6]. Overall, polycystic ovaries are observed in $20 \%$ to $30 \%$ of the population. It remains controversial whether the isolated presence of polycystic ovaries is clinically significant [1]. Nonetheless, asymptomatic women with polycystic ovaries have some abnormalities related to androgen secretion and insulin sensitivity [24].

To date, the precise mechanism(s) responsible for the development of polycystic ovaries is poorly understood. The potential contribution of the peripheral sympathetic nervous system to polycystic ovaries has been suggested by several observations, including the increased density of catecholaminergic nerves detected by histofluorescence in patients suffering from the disease [25] and the effectiveness of ovarian wedge resection in restoring ovulatory capacity, especially when the hilum, where nerves enter the ovary, is compromised [26].

The development and function of ovarian sympathetic innervations depend on the ability of the ovary to produce NGF [27]. Although ovarian NGF facilitates follicular development and ovulation, an excess of the neurotrophin in the rodent ovary reduces ovulatory capacity and causes the development of precystic follicles [15]. Our findings, which demonstrated an association between EV-induced cystic ovarian morphology and increased NGF expression, as defined by Western blot analysis, are consistent with previous observations [15]. Very recently, it was reported that ovarian production of NGF is increased in PCOS patients and transgenic overexpression of the neurotrophin targeted to the mouse ovary results in polycystic ovaries [14]. The previous and present results suggest that the overproduction of ovarian NGF is an important contributing factor to polycystic ovaries in both humans and rodents.

Our previous study could not exclude the competitive antagonism of KRGE against EV on estrogen receptors. As the ginsenosides share a backbone similar to steroidal hormones, some ginsenosides have been shown to bind to the same target protein as steroidal hormones, including estrogen receptors [28]. The present study suggests something beyond direct antagonism against $\mathrm{EV}$ because KRGE was administered beginning from 60 days after EV injection. We do not currently understand the molecular mechanisms for this apparent partial attenuation of NGF expression and reversal of polycystic ovaries in EV-induced rats following the administration of KRGE. These findings need to be further investigated in rela- tion to adrenoreceptors and neutrophin receptors, since they are possible participants in the development of EVinduced polycystic ovaries [29].

In summary, the present study using an EV-induced PCOS rat model demonstrates that KRGE decreases the ovarian production of NGF and cyst formation, even after polycystic ovaries are fully developed. These findings suggest that ovarian NGF contributes to the pathophysiology of polycystic ovaries and that established polycystic ovaries can be reversed by KRGE administration.

\section{ACKNOWLEDGEMENTS}

This work was supported by the 2006 grant from the Korean Society of Ginseng funded by Korea Ginseng Corporation.

\section{REFERENCES}

1. Azziz R, Carmina E, Dewailly D, Diamanti-Kandarakis E, Escobar-Morreale HF, Futterweit W, Janssen OE, Legro RS, Norman RJ, Taylor AE et al. The Androgen Excess and PCOS Society criteria for the polycystic ovary syndrome: the complete task force report. Fertil Steril 2009;91:456-488.

2. Goodarzi MO, Dumesic DA, Chazenbalk G, Azziz R. Polycystic ovary syndrome: etiology, pathogenesis and diagnosis. Nat Rev Endocrinol 2011;7:219-231.

3. Dumesic DA, Padmanabhan V, Abbott DH. Polycystic ovary syndrome and oocyte developmental competence. Obstet Gynecol Surv 2008;63:39-48.

4. Juengel JL, McNatty KP. The role of proteins of the transforming growth factor-beta superfamily in the intraovarian regulation of follicular development. Hum Reprod Update 2005;11:143-160.

5. Azziz R. Androgen excess is the key element in polycystic ovary syndrome. Fertil Steril 2003;80:252-254.

6. Mortensen M, Rosenfield RL, Littlejohn E. Functional significance of polycystic-size ovaries in healthy adolescents. J Clin Endocrinol Metab 2006;91:3786-3790.

7. Rosenfield RL. Clinical review: identifying children at risk for polycystic ovary syndrome. J Clin Endocrinol Metab 2007;92:787-796.

8. Sverrisdottir YB, Mogren T, Kataoka J, Janson PO, Stener-Victorin E. Is polycystic ovary syndrome associated with high sympathetic nerve activity and size at birth? Am J Physiol Endocrinol Metab 2008;294:E576-E581.

9. Heider U, Pedal I, Spanel-Borowski K. Increase in nerve fibers and loss of mast cells in polycystic and postmenopausal ovaries. Fertil Steril 2001;75:1141-1147. 
10. Brawer JR, Munoz M, Farookhi R. Development of the polycystic ovarian condition (PCO) in the estradiol valerate-treated rat. Biol Reprod 1986;35:647-655.

11. Lara HE, Dissen GA, Leyton V, Paredes A, Fuenzalida H, Fiedler JL, Ojeda SR. An increased intraovarian synthesis of nerve growth factor and its low affinity receptor is a principal component of steroid-induced polycystic ovary in the rat. Endocrinology 2000;141:1059-1072.

12. Pak SC, Lim SC, Nah SY, Lee J, Hill JA, Bae CS. Role of Korean red ginseng total saponins in rat infertility induced by polycystic ovaries. Fertil Steril 2005;84:1139-1143.

13. Pak SC, Kim SE, Oh DM, Shim KM, Jeong MJ, Lim SC, Nah SY, Park SH, Kang SS, Moon CJ et al. Effect of Korean red ginseng extract in a steroid-induced polycystic ovary murine model. Arch Pharm Res 2009;32:347-352.

14. Dissen GA, Garcia-Rudaz C, Paredes A, Mayer C, Mayerhofer A, Ojeda SR. Excessive ovarian production of nerve growth factor facilitates development of cystic ovarian morphology in mice and is a feature of polycystic ovarian syndrome in humans. Endocrinology 2009; 150:2906-2914.

15. Lara HE, Ferruz JL, Luza S, Bustamante DA, Borges Y, Ojeda SR. Activation of ovarian sympathetic nerves in polycystic ovary syndrome. Endocrinology 1993;133:26902695.

16. Lobos E, Gebhardt C, Kluge A, Spanel-Borowski K. Expression of nerve growth factor (NGF) isoforms in the rat uterus during pregnancy: accumulation of precursor proNGF. Endocrinology 2005;146:1922-1929.

17. Mathur R, Alexander CJ, Yano J, Trivax B, Azziz R. Use of metformin in polycystic ovary syndrome. Am J Obstet Gynecol 2008;199:596-609.

18. Yildiz BO. Oral contraceptives in polycystic ovary syndrome: risk-benefit assessment. Semin Reprod Med 2008; 26:111-120.

19. Panidis D, Georgopoulos NA, Piouka A, Katsikis I, Saltamavros AD, Decavalas G, Diamanti-Kandarakis E. The impact of oral contraceptives and metformin on antiMullerian hormone serum levels in women with polycys- tic ovary syndrome and biochemical hyperandrogenemia. Gynecol Endocrinol 2010; Epub ahead of print.

20. Bayrak A, Terbell H, Urwitz-Lane R, Mor E, Stanczyk FZ, Paulson RJ. Acute effects of metformin therapy include improvement of insulin resistance and ovarian morphology. Fertil Steril 2007;87:870-875.

21. Somunkiran A, Yavuz T, Yucel O, Ozdemir I. AntiMullerian hormone levels during hormonal contraception in women with polycystic ovary syndrome. Eur J Obstet Gynecol Reprod Biol 2007;134:196-201.

22. Ehrmann DA. Polycystic ovary syndrome. N Engl J Med 2005;352:1223-1236.

23. Norman RJ, Dewailly D, Legro RS, Hickey TE. Polycystic ovary syndrome. Lancet 2007;370:685-697.

24. Clayton RN, Ogden V, Hodgkinson J, Worswick L, Rodin DA, Dyer S, Meade TW. How common are polycystic ovaries in normal women and what is their significance for the fertility of the population? Clin Endocrinol (Oxf) 1992;37:127-134.

25. Semenova II. Adrenergic innervation of ovaries in SteinLeventhal syndrome. Vestn Akad Med Nauk SSSR 1969;24:58-62.

26. Katz M, Carr PJ, Cohen BM, Millar RP. Hormonal effects of wedge resection of polycystic ovaries. Obstet Gynecol 1978;51:437-444.

27. Dees WL, Hiney JK, Schultea TD, Mayerhofer A, Danilchik M, Dissen GA, Ojeda SR. The primate ovary contains a population of catecholaminergic neuron-like cells expressing nerve growth factor receptors. Endocrinology 1995; 136:5760-5768.

28. Gao QG, Chen WF, Xie JX, Wong MS. Ginsenoside Rg1 protects against 6-OHDA-induced neurotoxicity in neuroblastoma SK-N-SH cells via IGF-I receptor and estrogen receptor pathways. J Neurochem 2009;109:1338-1347.

29. Manni L, Holmang A, Lundeberg T, Aloe L, StenerVictorin E. Ovarian expression of alpha (1)- and beta (2)-adrenoceptors and p75 neurotrophin receptors in rats with steroid-induced polycystic ovaries. Auton Neurosci 2005;118:79-87. 